\title{
Lipid Clinics Network. Rationale and design of the EAS global project
}

\author{
Asiiat S. Alieva ${ }^{\text {a, e, }}$, Lale Tokgözoğlu ${ }^{\text {b }}$, Kausik K. Ray ${ }^{c}$, Alberico L. Catapano ${ }^{\mathrm{d}}$ \\ a Centre of Atherosclerosis and Lipid Disorders, Almazov National Medical Research Centre, Saint Petersburg, Russia \\ ${ }^{\mathrm{b}}$ Department of Cardiology, Hacettepe University, Ankara, Turkey \\ ${ }^{\mathrm{c}}$ Imperial Centre for Cardiovascular Disease Prevention, Department of Primary Care and Public Health, Imperial College, London, UK \\ d Department of Pharmacological and Biomolecular Sciences, University of Milano, Multimedica IRCCS, Milano, Italy \\ e Department of Pharmacological and Biomolecular Sciences (DisFeB), University of Milan, Milan, Italy
}

\section{A R T I C L E I N F O}

Article history:

\section{Keywords:}

Cardiovascular risk

Dyslipidemia

Risk management

European guidelines

\begin{abstract}
A B S T R A C T
The evidence of the causal role of low-density lipoprotein cholesterol in the development of atherosclerotic cardiovascular disease is well-established. The clearly identified common position of the European guidelines proclaims necessity to decrease LDL-C concentrations based on a proper risk stratification. However, current worldwide situation with the lipid management still demonstrates inadequate dyslipidemia control, that is probably related to a healthcare system issues. As the need to standardize and implement approaches following the guidelines into clinical practice remains a challenge, the EAS initiates the Lipid Clinics Network project, aiming to provide a structure to establish uniform EU-wide standards of diagnosis, management and treatment of patients with lipid disorders, based on the ESC/EAS Guidelines on management of dyslipidaemias.
\end{abstract}

( 2021 The Authors. Published by Elsevier B.V. This is an open access article under the CC BY-NC-ND license (http://creativecommons.org/licenses/by-nc-nd/4.0/).

\section{Introduction}

Despite the evidence of the causal role of low-density lipoprotein cholesterol (LDL-C) in the development of atherosclerotic cardiovascular (CV) disease (CVD) and the clearly identified common position of the European guidelines proclaiming necessity to decrease LDL-C concentrations based on a proper risk stratification, current worldwide situation with the lipid management still demonstrates inadequate control of mentioned CV risk factor, which probably indicates a range of difficulties at health system practices. The results of the recent study of lipid-modifying therapy use in primary and secondary care DA VINCI emphasized the gap between clinical guidelines and clinical practice based on the fact that only 33\% of patients achieved their risk-based 2019 goal. Furthermore, within the risk groups structure $17 \%$ and $22 \%$ for very high-risk primary and secondary prevention patients, respectively were on 2019 targets [1].

Problematic issues are related to

\footnotetext{
* Corresponding author.

E-mail address: alieva_as@almazovcentre.ru (A.S. Alieva).
}

- difficulties in the accuracy and timeliness of CV risk stratification in patients, predominantly in the framework of primary prevention

- providing continuity in the management of patients with dyslipidemias between specialized centers and GP-practitioners, and, as a result, failure in achieving LDL-C target values

- patient adherence to lipid-lowering therapy.

Along with the necessity of using current risk assessment tools new approaches should be reviewed to increase its accuracy. Based on the genetic studies utilizing the Mendelian randomization approach the risk assessment paradigm moved the focus to the rationale of an early risk identification and intervention according to the importance of lifetime risk [2]. In perspectives in order to decrease the financial burden to national healthcare systems and to preserve the health of nations the ideology of intervention should be based on the lifetime risk assessment within the primary prevention area instead of short-time risk ones only [3]. Moving forward along with the well-established scientific background of these concepts there is a need to provide practical healthcare system with concrete steps and validated tools for using in a real clinical practice. First on line calculator based on the lifetimeperspective cardiovascular disease (LIFE-CVD) model for the estimation of treatment-effects cholesterol-lowering, blood pressure lowering antithrombotic therapy, and smoking cessation in 
apparently healthy people was presented in 2019 [4].

Talking about the management of specific high risk groups patients a range of successful steps have been done. Since 2015, the European Atherosclerosis Society (EAS) project, the EAS FH Studies Collaboration, has been successfully in operation, aiming consolidation of the efforts to identify and to treat patients with familial hypercholesterolaemia (FH) that belong to high or very high $\mathrm{CV}$ risk. The maintenance of a register allowed to draw attention to this problem and significantly increase the percentage of FH detection. The six-year results of the project provided the registry with more than 62,000 patients from 62 countries, among which 41,000 patients are participants with a definite or probable diagnosis according to clinical and/or genetic FH criteria.

Nevertheless, while a proper management of high and very high $\mathrm{CV}$ risk groups is relatively fast and effective within the short time period, the only way to change the curve of morbidity and mortality on a population level is to use also a non-high CV risk management strategy. Given the fact that according to the Rose paradox [5] at the population level, the largest number of cardiovascular events occurs in the area of non-high CV risk patients due to its multitude, there is an obvious necessity to improve the system of dyslipidaemia management for all risk groups, and, even more important, to build a clear system of integration between lipid clinics, playing a role of competence centers for the establishing dyslipidemia management EU-standards within primary health care structures, i.e. GP practitioners. An increase of attention to the development of optimal integrated primary care is related to the necessity to win through the lack of resources, long waiting time for a consultation and the lack of training. A comprehensive service within primary care and the involvement of multidisciplinary team using EUstandards of dyslipidemia treatment seems a key step in nonhigh risk patients management. Informatics-based clinical decision support systems may serve as a tool for an increase of physician adherence to best practices in dyslipidemia treatment recommendations.

All the issues mentioned above demonstrate the need to standardize approaches following the guidelines. Therefore, the EAS initiated the Lipid Clinics Network project, aiming to provide a structure to establish uniform EU-wide standards of diagnosis, management and treatment of patients with lipid disorders, based on the ESC/EAS Guidelines on management of dyslipidaemias.

\section{Key objectives}

- to set up, harmonise and structure a network of lipid clinics, leveraging on the diverse connections between leading lipid clinics in Europe via the Society and its members, FHSC and national atherosclerosis societies and through exchange through the network

- to update existing clinics to specified standards, and establish clinics in countries where none exist at present

- to establish EU-wide standard procedures for diagnosis \& treatment of lipid disorders, with regular updates and standardisation of existing procedures, according to current European Guidelines on management of dyslipidaemias

- to establish EU-wide common standards \& methods, and use of standardised measurements \& software (update current standards, establish standards in countries where none exist at present)

- to establish standard recommendations in EU-wide public health policies regarding the diagnosis (with \& without genetic testing) and treatment of lipid disorders.

- to promote awareness among policy makers within governments and national health funds in each EU country, driving for higher financing for research and educational programmes and reimbursement of therapies dedicated to patients with lipid disorders.

\section{Key steps, on a country-by-country basis}

- review current practice: through a survey of stakeholders from all aspects of lipid disorder patient diagnosis and care, build a base-line picture of how (or whether) current guidelines in management of dyslipidaemias are put into practice

- identify gaps in knowledge and/or processes: use the responses to the survey to identify priority areas where action is needed so that practice is as recommended in the guidelines

- address gaps in knowledge and/or processes: design educational and training activities, procedure review etc., bringing in expertise from throughout the network to share best practice

- evaluate outcomes: for the training and educational activities carried out, compare practice post-activity vs. pre activity. How is patient diagnosis and care improved? Which measures are most effective?

- share best practice: successful outcomes can be fed back into the network to help other countries

\section{Benefits}

By forming a structured European network of clinics dedicated to diagnosis and management of lipid disorders, we will facilitate exchange, educate and promote the work of clinical lipidologists, enabling them to share:

- clinical experiences in the management of affected patients

- knowledge about diagnosis

- information about outcomes

- approaches to barriers to sub-optimal patient care

The Lipid Clinics Network will also serve as the basis for educational activities and training, including web-based and online education.

\section{Conclusion}

Dyslipidemia is one of the key modifiable risk factor of atherosclerotic cardiovascular disease that brings one of the biggest impact into the structure of morbidity and mortality all over the Europe. Prevention and treatment programmes are widely used in order to preserve health on a population level, however, successful implementation of the current guidelines into real clinical practice remains a challenge. The Lipid Clinics Network aims to provide a structure to establish uniform EU-wide standards of diagnosis, management and treatment of patients with lipid disorders.

\section{CRediT authorship contribution statement}

Asiiat S. Alieva: Writing - original draft. Lale Tokgözoğlu: Conceptualization, Writing - review \& editing. Kausik K. Ray: Conceptualization, Writing - review \& editing. Alberico L. Catapano: Conceptualization, Writing - review \& editing.

\section{Declaration of competing interest}

None relevant to the work described in this manuscript. 


\section{Acknowledgments}

The work of ALC has been supported by Ministry of Health Ricerca Corrente - IRCCS MultiMedica, PRIN 2017H5F943 and ERANET ER-2017-2364981. This article is part of a Supplement entitled "Plasma lipids and cardiovascular risk: Nutritional and therapeutic approaches" published with support from Società Italiana di Terapia Clinica e Sperimentale (SITeCS).

\section{References}

[1] Ray KK, Molemans B, Schoonen WM, Giovas P, Bray S, et al. EU-wide crosssectional observational study of lipid-modifying therapy use in secondary and primary care: the DA VINCI study. Eur J Prev Cardiol. ISSN 2020. https:// doi.org/10.1093/eurjpc/zwaa047. 2047-4873.

[2] Ference BA, Ginsberg HN, Graham I, Ray KK, Packard CJ, et al. Low-density lipoproteins cause atherosclerotic cardiovascular disease. 1. Evidence from genetic, epidemiologic, and clinical studies. A consensus statement from the European Atherosclerosis Society Consensus Panel. Eur Heart J 2017;38: 2459-72. https://doi.org/10.1093/eurheartj/ehx144.

[3] K Ray K, Laufs U, Cosentino F, Lobo MD, Landmesser U. The year in cardiology: cardiovascular prevention: the year in cardiology 2019. Eur Heart J 2020;41: 1157-63. https://doi.org/10.1093/eurheartj/ehz929.

[4] Jaspers NEM, Blaha MJ, Matsushita K, van der Schouw YT, Wareham NJ, et al. Prediction of individualized lifetime benefit from cholesterol lowering, blood pressure lowering, antithrombotic therapy, and smoking cessation in apparently healthy people. Eur Heart J 2020;41:1190-9. https://doi.org/10.1093/ eurheartj/ehz239.

[5] Rose G. The strategy of preventive medicine. England: Oxford University Press: 1992. p. 171. 